\title{
THE GAUSS-BONNET THEOREM AND THE TAMAGAWA NUMBER
}

\author{
BY TAKASHI ONO
}

Communicated by G. D. Mostow, September 14, 1964

This note is an outline of some of the author's recent work on the Tamagawa numbers. Our purpose is to express the Tamagawa number of Chevalley groups in terms of Euler-Poincaré characteristics, Bernoulli numbers and Gaussian curvatures.

The method can, of course, be applied to derive other formulas of the same type. We treat, however, only Chevalley groups, because it is in the present case that the main ideas of our method are most clear. The details and further results will be given elsewhere.

Let $G_{0}$ be a connected semisimple real Lie group without compact factors and center, $\mathfrak{g}_{0}$ its Lie algebra and put $\mathfrak{g}=\mathfrak{g}_{0} \otimes C$. Let $K$ be a maximal compact subgroup of $G_{0}$ and let be its Lie algebra. To the Cartan decomposition $\mathfrak{g}_{0}=\mathfrak{f}+\mathfrak{p}, \mathfrak{p}$ being the orthogonal complement of $\mathfrak{l}$ with respect to the Killing form $B$ of $\mathfrak{g}$, there corresponds the dual $\mathfrak{u}=\mathfrak{f}+(-1)^{1 / 2} \mathfrak{p}$. Denote by $U$ the connected compact group whose Lie algebra is $\mathfrak{u}$ and by $T$ a maximal torus of $U$. We denote by $d G_{0}, d U$, $d K, d T, d\left(G_{0} / K\right), d(U / K), d(U / T)$ the volume elements corresponding to the Riemannian structure on each space obtained naturally by $B$ (cf. [4, Chapter IV]). We also denote by $\kappa(M)$ the Gaussian curvature of a Riemannian manifold $M$ and by $\chi(M)$ the Euler-Poincaré characteristic. One has $\kappa(U / K)=(-1)^{m / 2} \kappa\left(G_{0} / K\right)$ with $m=\operatorname{dim}\left(G_{0} / K\right)$. By the Gauss-Bonnet theorem (cf. [1], [2]), we have

$$
\begin{aligned}
& \frac{2 \kappa(U / K)}{A_{m}} \int_{U / K} d(U / K)=\chi(U / K), \\
& \frac{2 \kappa(U / T)}{A_{2 N}} \int_{U / T} d(U / T)=\chi(U / T)=[W],
\end{aligned}
$$

where $A_{m}$ is the surface area of the unit $m$-sphere, $N$ is the number of positive roots of $\mathfrak{g}$ and $[W]$ is the order of the Weyl group $W$ of $\mathfrak{g}$. Eliminating the volume of $U$ from (1), (2), one gets

$$
\int_{K} d K=\int_{T} d T \frac{A_{2 N}[W]_{\kappa}(U / K)}{A_{m} \chi(U / K)_{\kappa}(U / T)} .
$$

Let $\Gamma$ be a discrete subgroup of $G_{0}$ such that 


$$
\int_{\Gamma \backslash G_{0}} d G_{0}<+\infty
$$

We define the "Euler-Poincaré characteristic" of $\Gamma \backslash G_{0} / K$ by

$$
\frac{2 \kappa\left(G_{0} / K\right)}{A_{m}} \int_{\Gamma \backslash G_{0} / K} d\left(G_{0} / K\right)=\chi\left(\Gamma \backslash G_{0} / K\right) .
$$

From (3), (4), we have

$$
\int_{\Gamma \backslash G_{0}} d G_{0}=(-1)^{m / 2} \int_{T} d T \frac{A_{2 N}[W] \chi\left(\Gamma \backslash G_{0} / K\right)}{2 \chi(U / K)_{\kappa}(U / T)} .
$$

Now, let $G \subset \mathrm{GL}(n)$ be a connected semisimple algebraic group defined over $Q$. Let $\mathrm{g}$ be the Lie algebra of $G$ and let $X_{i}, 1 \leqq i \leqq n$, be a basis of $\mathfrak{g}$ rational over $Q$. Call $\omega$ the algebraic $n$-form over $Q$ which is the product of 1 -forms dual to $X_{i}$. Let $G_{0}$ be the identity component of $G_{R}$, and let $g_{0}$ be the Lie algebra of $G_{0}$. Then $\omega$ induces on $G_{0}$ a volume element $\omega_{R}$, and we see that

$$
d G_{0}=\left(\operatorname{det} B^{+}\left(X_{i}, X_{j}\right)\right)^{1 / 2} \omega_{R},
$$

where $B^{+}(X, Y)=-B(X, \sigma Y), \sigma$ being the conjugation of $g$ with respect to $\mathfrak{u}$.

Assume now that $G$ is the Chevalley group, i.e., the identity component of the group of automorphisms of a complex semisimple Lie algebra $\mathfrak{g}$. With respect to a Chevalley basis of $\mathfrak{g}$ (cf. [3, Théorème 1]) $G$ becomes an algebraic subgroup of $\mathrm{GL}(n)$ defined over $\boldsymbol{Q}, n=\operatorname{dim} \mathfrak{g}$. By choosing suitable $K$ and $T$, and putting $\Gamma=G_{\boldsymbol{Z}} \cap G_{0}$, here $G_{\boldsymbol{Z}}$ being the group of units of $G$, we get from (5), (6) the following:

$$
\int_{G_{Z} \backslash G_{R}} \omega_{R}=\int_{\Gamma \backslash G_{0}} \omega_{R}
$$

$$
=\frac{(-1)^{(l+N) / 2} 2^{l+N} N ![W]}{(2 N) ! C} \prod_{r>0}\langle r, r\rangle \frac{\chi\left(\Gamma \backslash G_{0} / K\right)}{\chi(U / K)_{\kappa}(U / T)} \pi^{l+N},
$$

where $\langle r, r\rangle$ is the inner product defined by $B, C$ is the determinant of the Cartan matrix and $l$ is the rank of $\mathfrak{g}$.

On the other hand, $\omega$ induces a measure $\omega_{p}$ on $G_{Q_{p}}$ for all prime numbers $p$. Denote by $G z_{p}$ the group of $p$-adic units of $G_{Q_{p}}$. By the results of Iwahori-Matsumoto (cf. [5, Corollary 2.17]) and Ree (unpublished) on $p$-adic Chevalley groups, we get 


$$
\int_{G_{Z_{p}}} \omega_{p}=\frac{1}{|C|_{p}} \frac{\left[G_{F_{p}}\right]}{p^{n}} \text { for all } p
$$

where $G_{F_{p}}$ is the finite group of rational points over $F_{p}$. Since $\Pi_{v}|C|_{v}$ $=1$, we get

$$
\prod_{p} \int_{G_{Z_{p}}} \omega_{p}=C \prod_{i=1}^{l} \zeta\left(a_{i}\right)^{-1}
$$

where the $a_{i}$ are integers such that the Poincare polynomial of the compact form of $G$ is $\prod_{i=1}^{l}\left(t^{2 a_{i}-1}+1\right)$ (cf. [3, p. 46]). When $a_{i}$ are all even, we have

$$
\zeta\left(a_{i}\right)=2^{a_{i}-1} \frac{\pi^{a_{i}}}{a_{i} !} B_{a_{i} / 2} \quad(1 \leqq i \leqq l) .
$$

Since $\sum_{i=1}^{l} a_{i}=l+N$ and the class number of our $G$ is 1 , we get, from (7) and (8), the following:

(9) $\tau(G)=\frac{(-1)^{(l+N) / 2} 2^{l} N ![W]}{(2 N) !} \prod_{i=1}^{l} a_{i} ! B_{a_{i} / 2}^{-1} \prod_{r>0}\langle r, r\rangle \frac{\chi\left(\Gamma \backslash G_{0} / K\right)}{\chi(U / K)_{\kappa}(U / T)}$,

with $\Gamma=G_{\boldsymbol{Z}} \cap G_{0}$.

REMARK 1. Our method does not apply if $m$ is odd or $\chi(U / K)=0$ or $a_{i}$ is odd for some $i$. When $\mathfrak{g}$ is simple, these happen for groups of type $A_{l}(l \geqq 2), D_{l}$ (lodd) and $E_{6}$ (cf. [3, p. 64]). The first two cases are classical and the $\tau(G)$ are known (cf. [9]). Therefore $E_{6}$ is the only exception among Chevalley groups. It is interesting to notice that $\chi(U / K) \neq 0$ if and only if $a_{i}$ are all even. This would explain the difficulty of finding $\zeta(2 n+1)$.

REMARK 2. On the right-hand side of (9), all factors except $\chi\left(\Gamma \backslash G_{0} / K\right)$ are $\in Q$. As for $\chi\left(\Gamma \backslash G_{0} / K\right)$, one could probably prove (even for the general case) that $\chi\left(\Gamma^{\prime} \backslash G_{0} / K\right)$ is $\in \boldsymbol{Z}, \Gamma^{\prime}$ being a normal subgroup of $\Gamma$ with finite index, by making use of Allendoerfer-Weil's theorem as Satake has done for symplectic groups (cf. [1], [6]).

REMARK 3. For general semisimple groups, the difficulty arises from the $p$-adic part rather than from the $\infty$-adic part. Namely, the zeta function should be replaced by $L$-functions and one has to know their values at some integers.

\section{REFERENCES}

1. C. B. Allendoerfer and A. Weil, The Gauss-Bonnet theorem for Riemannian polyhedra, Trans. Amer. Math. Soc. 53 (1943), 101-129.

2. S. S. Chern, A simple intrinsic proof of the Gauss-Bonnet formula for closed Riemannian manifolds, Ann. of Math. (2) 45 (1944), 747-752. 
3. C. Chevalley, Sur certains groupes simples, Tôhoku Math. J. 7 (1955), 14-66.

4. S. Helgason, Differential geometry and symmetric spaces, Academic Press, New York, 1962.

5. N. Iwahori and $\mathrm{H}$. Matsumoto, On some Bruhat decompositions and the structure of the Hecke rings of p-adic Chevalley groups (to appear).

6. I. Satake, The Gauss-Bonnet Theorem for V-manifolds, J. Math. Soc. Japan 9 (1957), 464-492.

7. C. L. Siegel, Symplectic geometry, Amer. J. Math. 65 (1943), 1-86.

8. A. Weil, Adèles et groupes algébriques, Séminaire Bourbaki, 1958/59, Exp. 186, Secrétariat Mathématique, Paris, 1959.

9. - Adeles and algebraic groups, Lecture Notes, Institute for Advanced Study, Princeton, N. J., 1961.

University of Pennsylvania 